\title{
Discovery of an energetic bipolar molecular outflow towards IRAS 16547-4247
}

\author{
G. Garay ${ }^{1}$, D. Mardones ${ }^{1}$, L. Bronfman ${ }^{1}$, K. J. Brooks ${ }^{2}$, L. F. Rodríguez ${ }^{3}$, R. Güsten ${ }^{4}$, L.-Å. Nyman ${ }^{5}$, \\ R. Franco-Hernández ${ }^{6}$, and J. M. Moran ${ }^{6}$ \\ 1 Departamento de Astronomía, Universidad de Chile, Casilla 36-D, Santiago, Chile \\ e-mail: guido@das.uchile.cl \\ 2 Australia Telescope National Facility, PO Box 76, Epping 1710 NSW, Australia \\ 3 Centro de Radioastronomía y Astrofísica, UNAM, Apdo. Postal 3-72, Morelia, Michoacán, 58089, México \\ ${ }^{4}$ Max-Planck-Institut für Radioastronomie, Auf dem Hügel 69, 53121 Bonn, Germany \\ 5 European Southern Observatory, Casilla 19001, Santiago, Chile \\ ${ }^{6}$ Harvard-Smithsonian Center for Astrophysics, 60 Garden Street, Cambridge, MA 02138, USA
}

Received 30 March 2006 / Accepted 16 November 2006

\section{ABSTRACT}

\begin{abstract}
Aims. We present molecular line observations of the molecular core associated with IRAS 16547-4247, which have allowed us to determine its physical and kinematical properties at angular resolutions of $\sim 18^{\prime \prime}$.

Methods. The observations were made using the Atacama Pathfinder Experiment Telescope in the $J=3 \rightarrow 2$ transitions of ${ }^{12} \mathrm{CO}$ and ${ }^{13} \mathrm{CO}, J=4 \rightarrow 3$ transitions of $\mathrm{HCO}^{+}$and $\mathrm{H}^{13} \mathrm{CO}^{+}$, and $J=7 \rightarrow 6$ transition of CS.

Results. Our observations reveal the presence of a collimated bipolar outflow with lobes $\sim 0.7 \mathrm{pc}$ in extent and aligned with the thermal jet located at the center of the core. The morphology and velocity structure of the flow are well described by a biconical outflow that is inclined from the line of sight by an angle of $84^{\circ}$, has a semi-opening angle of $14^{\circ}$, and in which the gas moves outwards with a constant total velocity, with respect to the cone apex, of $\sim 120 \mathrm{~km} \mathrm{~s}^{-1}$. The outflow is massive and energetic (flow mass $\sim 110 M_{\odot}$; mass outflow rate $\sim 2 \times 10^{-2} M_{\odot} \mathrm{yr}^{-1}$; momentum $\sim 2 \times 10^{3} M_{\odot} \mathrm{km} \mathrm{s}^{-1}$ and kinetic energy $\sim 9 \times 10^{47} \mathrm{erg}$ ), and has a dynamical time scale of $6 \times 10^{3} \mathrm{yr}$. These parameters are consistent with the flow being driven by a young massive stellar object with $L_{\mathrm{bol}} \sim 6 \times 10^{4} L_{\odot}$.
\end{abstract}

Key words. ISM: jets and outflows - ISM: molecules - stars: formation

\section{Introduction}

One of the major questions related to the formation of stars is whether or not the successful model of low-mass star formation, based on accretion via a circumstellar disk and collimated outflow in the form of a jet (Shu et al. 1987), can be extended to high-mass stars. Support for a similar mechanism of formation is provided by the fact that molecular outflows have been found to be driven by protostellar objects with a broad range of masses (Zhang et al. 2001; Beuther et al. 2002c). The molecular mass outflow rate and the luminosity of the driving source are strongly correlated (Shepherd \& Churchwell 1996; Churchwell 2000), which suggests that there is a strong link between accretion and outflow for a wide range of luminosities. There are, however, only a few cases of luminous $\left(L>4 \times 10^{4} L_{\odot}\right)$ massive young stellar objects (O-type protostars) known to be associated with collimated bipolar outflows. Whether this is an intrinsic property of the most massive stars or due to an observational selection effect - massive stars are rarer and their evolutionary time scales are much shorter than those of low mass stars - has yet to be established.

A wealth of observations of molecular line emission in high density tracers (e.g., Plume et al. 1992; Juvela 1996; Plume et al. 1997) and of dust continuum emission (e.g., Beuther et al. 2002b; Faúndez et al. 2004) show that massive stars $(M>$ $8 M_{\odot}$ ) form in regions of molecular gas with distinctive physical parameters, namely radii of typically $\sim 0.4 \mathrm{pc}$, densities of $\sim 5 \times 10^{5} \mathrm{~cm}^{-3}$ and masses of $\sim 4 \times 10^{3} M_{\odot}$. Hereinafter we will refer to these structures as massive and dense molecular cores. Most massive and dense cores are located within GMC's and in complex environments, which makes the observational study of the early stages of massive star formation difficult. There are, however, a few massive and dense isolated cores, which due to their simple geometry and environment, may prove to be ideal cases for a detailed study of the process of formation of individual massive stars.

G343.126-0.062 is an isolated massive and dense core located at a distance of $2.9 \mathrm{kpc}$ (Garay et al. 2003). It is also known as IRAS 16547-4247 (Bronfman et al. 1996) which has a bolometric luminosity of $6.2 \times 10^{4} L_{\odot}$, equivalent to that of a single 08 zero-age main-sequence star. The core mass derived from observations of the dust continuum emission at $1.2 \mathrm{~mm}$ is $1.3 \times 10^{3} M_{\odot}$. Radio continuum observations show three sources, near the center of the core, aligned roughly in a north-south direction (Garay et al. 2003; Rodríguez et al. 2005). The central object is a thermal radio jet, while the two outer lobes, symmetrically separated from the central source by an angular distance of $\sim 10^{\prime \prime}$, are radio $\mathrm{HH}$ objects. The position angle of the radio lobes is $163 \pm 1^{\circ}$ (Rodríguez et al. 2005). The jet is closely aligned with the outer lobes and has an opening angle of $\sim 25^{\circ}$. Brooks et al. (2003) reported a chain of $\mathrm{H}_{2} 2.12 \mu \mathrm{m}$ emission knots that trace a collimated flow extending over $1.5 \mathrm{pc}$ that emanates from close to the central component of the triple radio source and has a position angle similar to that defined by the 
Table 1. Summary of parameters for APEX observations.

\begin{tabular}{lccccccc}
\hline \hline Line & $\begin{array}{c}\text { Frequency } \\
(\mathrm{MHz})\end{array}$ & $\begin{array}{c}\text { Beam } \\
(F W H M)\end{array}$ & Spacing & $\begin{array}{c}\text { Positions } \\
\text { observed }\end{array}$ & $\begin{array}{c}t_{\text {in }} \\
(\mathrm{s})\end{array}$ & $\begin{array}{c}\Delta v \\
\left(\mathrm{~km} \mathrm{~s}^{-1}\right)\end{array}$ & $\begin{array}{c}\text { rms noise } \\
(\mathrm{K})\end{array}$ \\
\hline $\mathrm{CO}(3 \rightarrow 2)$ & 345795.991 & $18^{\prime \prime}$ & $20^{\prime \prime}$ & 40 & 40 & 0.1058 & 0.16 \\
${ }^{13} \mathrm{CO}(3 \rightarrow 2)$ & 330587.957 & $19^{\prime \prime}$ & $20^{\prime \prime}$ & 15 & 45 & 0.1107 & 0.42 \\
$\mathrm{CS}(7 \rightarrow 6)$ & 342882.950 & $18^{\prime \prime}$ & $20^{\prime \prime}$ & 9 & 80 & 0.1067 & 0.18 \\
$\mathrm{HCO}^{+}(4 \rightarrow 3)$ & 356734.250 & $17^{\prime \prime}$ & $20^{\prime \prime}$ & 9 & 80 & 0.1026 & 0.15 \\
$\mathrm{H}^{13} \mathrm{CO}^{+}(4 \rightarrow 3)$ & 346998.540 & $18^{\prime \prime}$ & $20^{\prime \prime}$ & 9 & 15 & 0.1055 & 0.42 \\
$\mathrm{C}^{17} \mathrm{O}(3 \rightarrow 2)$ & 337061.100 & $18^{\prime \prime}$ & - & 1 & 220 & 0.1085 & 0.14 \\
\hline
\end{tabular}

outer lobes of the triple radio source. Most likely this extended component traces gas ejected in the past by the central object of the triple source.

In this paper we present observations towards IRAS $16547-4247$ in molecular lines of ${ }^{12} \mathrm{CO},{ }^{13} \mathrm{CO}, \mathrm{HCO}^{+}$, $\mathrm{H}^{13} \mathrm{CO}^{+}$, and $\mathrm{CS}$, all of them in the $1 \mathrm{~mm}$ wavelength range, with angular resolutions of $\sim 18^{\prime \prime}$. We report the discovery of a highly energetic bipolar molecular outflow, centered on the thermal radio jet. The observed and derived characteristics of the outflow suggest that its driving source is a young luminous protostellar object, confirming that collimated outflows can also be present in the most massive protostars.

\section{Observations}

The observations were made during July 26-27, 2005, with the Atacama Pathfinder Experiment (APEX ${ }^{1}$ ) 12-m telescope 1ocated at Llano de Chajnantor in the Atacama desert of Chile. The observed transitions and basic observational parameters are summarized in Table 1. At the frequencies of the observed lines, the half-power beam width of the telescope is $\sim 18^{\prime \prime}$ and the main beam efficiency is 0.73 . A detailed description of APEX and its performance are given by Güsten et al. (2006). The frontend consisted of a single pixel heterodyne $\mathrm{SiS}$ receiver and the backend of a Fast Fourier Transform Spectrometer built by the MPIfR. A bandwidth of $1 \mathrm{GHz}$ with 8192 frequency channels was used, which provided a velocity coverage of $\sim 870 \mathrm{~km} \mathrm{~s}^{-1}$ and a velocity resolution of $\sim 0.11 \mathrm{~km} \mathrm{~s}^{-1}$ at $350 \mathrm{GHz}$. The observations were performed in position-switch mode and taken under good weather conditions. System temperatures were in the range $100-230 \mathrm{~K}$. The ${ }^{12} \mathrm{CO}(3 \rightarrow 2)$ emission was mapped in a region of $100^{\prime \prime} \times 160^{\prime \prime}$ in size. The integration time on source at each position was $40 \mathrm{~s}$, resulting in an rms noise of typically $0.16 \mathrm{~K}$ in antenna temperature. The ${ }^{13} \mathrm{CO}(3 \rightarrow 2)$ emission was mapped in a region of $60^{\prime \prime} \times 80^{\prime \prime}$ in size. The integration time on source at each position was $45 \mathrm{~s}$, resulting in an rms noise of typically $0.42 \mathrm{~K}$ in antenna temperature. The emission in the other transitions was mapped in a region of $60^{\prime \prime} \times 60^{\prime \prime}$ in size. All maps were centered on the position of the IRAS source $\left(\alpha=16^{\mathrm{h}} 58^{\mathrm{m}} 16.9^{\mathrm{s}} ; \delta=-42^{\circ} 52^{\prime} 7^{\prime \prime} \mathrm{J} 2000\right)$ and made with an angular spacing of $20^{\prime \prime}$. We also observed the $\mathrm{C}^{17} \mathrm{O}(3 \rightarrow 2)$ emission towards the peak position of the core. Uncertainties in the pointing accuracy are estimated at $3^{\prime \prime}$.

We also report here observations of the $\mathrm{HCO}^{+}(1 \rightarrow 0)$ line emission made with the 4-m NANTEN telescope located in Las Campanas, Chile, during January 2003. The $F W H M$ beam width and main beam efficiency at the frequency of the line $(89.18852 \mathrm{GHz})$ are $3.4^{\prime}$ and 0.89 , respectively. The front-end

1 This publication is based on data acquired with the Atacama Pathfinder Experiment (APEX). APEX is a collaboration between the Max-Planck-Institut für Radioastronomie, the European Southern Observatory, and the Onsala Space Observatory. was an SIS mixer receiver. We mapped, in position-switching mode, a region of $12^{\prime} \times 12^{\prime}$ with a sampling interval of $2^{\prime}$. The system temperatures, including the atmosphere, were typically 90-100 K (SSB) toward the zenith. The spectrometer was an acousto-optical spectrometer which provided a velocity resolution of $0.483 \mathrm{~km} \mathrm{~s}^{-1}$ and a velocity coverage of $500 \mathrm{~km} \mathrm{~s}^{-1}$, at the observed frequency. The integration time on source per position was $80 \mathrm{~s}$. The typical rms noise level of the spectra is $75 \mathrm{mK}$.

\section{Results}

\subsection{Molecular core}

We find that the emission from the core ambient gas, in all the molecular lines we mapped, arises from nearly circularly symmetric structures, as is illustrated in Figs. 1 and 2. Figure 1 shows maps of the velocity integrated ambient gas emission (velocity range from -36.1 to $\left.-23.9 \mathrm{~km} \mathrm{~s}^{-1}\right)$ in the $\operatorname{CS}(7 \rightarrow 6)$ and $\mathrm{HCO}^{+}(4 \rightarrow 3)$ lines observed with APEX and in the $\mathrm{CS}(5 \rightarrow 4)$ line observed with the 15-m SEST telescope located in La Silla, Chile. Figure 2 shows a map of the velocity integrated ambient gas emission in the $\mathrm{HCO}^{+}(1 \rightarrow 0)$ line observed with NANTEN. Note the large difference in angular scale between both figures. Table 2 gives the observed sizes determined from the maps using the task Gauss_2d of the GILDAS data analysis package. Also given in Table 2 are the sizes determined from previous observations made using SEST $\left(\mathrm{CS}(2 \rightarrow 1), \mathrm{CS}(5 \rightarrow 4)\right.$, and SO(6 $6_{5} \rightarrow$ 54) lines; Garay et al. 2003). Interestingly, we find that the observed size of the emitting region differs among the various transitions and is correlated with the beam size of the telescope used to observe the line. This is shown in Fig. 3 which shows the observed size $(F W H M)$ of the emission $\left(\theta_{\text {obs }}\right)$ versus the beam size $\left(\theta_{\text {beam }}\right)$ for all the available observations. A least squares fit to the observed relationship gives $\theta_{\text {obs }}=(1.59 \pm 0.05) \theta_{\text {beam }}$.

Figure 4 shows spectra observed toward the peak position of the IRAS $16547-4247$ core. The ${ }^{13} \mathrm{CO}(3 \rightarrow 2)$ and $\mathrm{HCO}^{+}(4 \rightarrow 3)$ spectra show, on one hand, double-peaked line profiles, with a bright blue-shifted peak and a weaker red-shifted peak. On the other hand, the profiles of the $\mathrm{C}^{17} \mathrm{O}(3 \rightarrow 2)$ and $\mathrm{H}^{13} \mathrm{CO}^{+}(4 \rightarrow 3)$ lines show a symmetric single component with a peak line center velocity of $\sim-30.6 \mathrm{~km} \mathrm{~s}^{-1}$. In addition, the relative minimum intensity (dip) of the optically thick line profiles occurs at a velocity that is higher than the peak velocity of the optically thin lines. These spectroscopic signatures suggest that the bulk of the molecular gas toward IRAS $16547-4247$ is undergoing largescale inward motions. Infalling motions traced by optically thick molecular lines are expected to produce line profiles showing blue asymmetry, whereas optically thin lines are expected to exhibit symmetrical profiles (Mardones et al. 1997). In particular, the shift of the dip to a velocity higher than the center-of-rest velocity of the cloud is a strong indication of the collapse of the outer layers (Snell \& Loren 1977). 


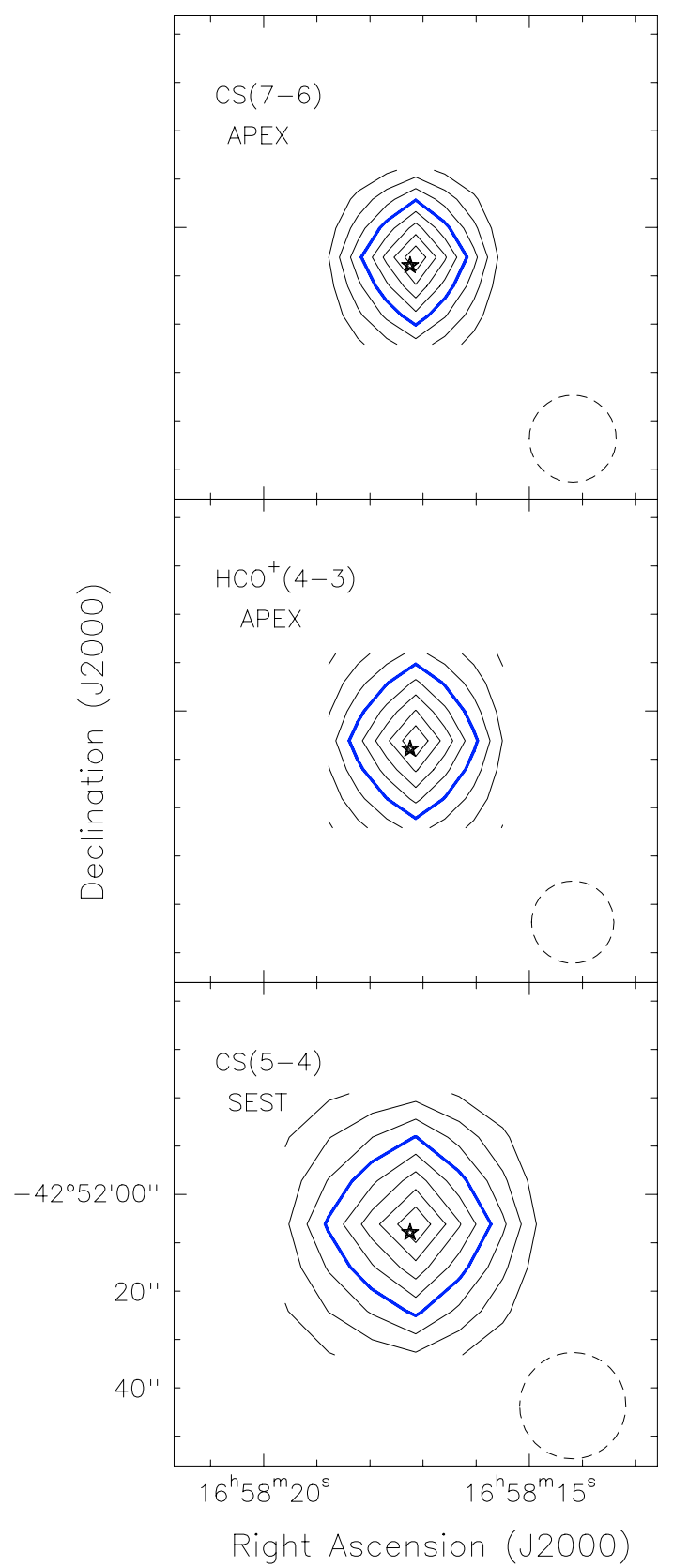

Fig. 1. Maps of the velocity integrated molecular line emission from the ambient gas of IRAS 16547-4247. The velocity range of integration is from -36.1 to $-23.9 \mathrm{~km} \mathrm{~s}^{-1}$. Contour levels are 20, 30, 40, 50, 60, 70, 80 , and $90 \%$ of the peak velocity integrated antenna temperature. The FWHM beam is shown in the lower right corner. The star denotes the position of the thermal radio jet. Top: $\mathrm{CS}(7 \rightarrow 6)$ emission observed with APEX. The peak is $5.34 \mathrm{~K} \mathrm{~km} \mathrm{~s}^{-1}$. Middle: $\mathrm{HCO}^{+}(4 \rightarrow 3)$ emission observed with APEX. The peak is $8.79 \mathrm{~K} \mathrm{~km} \mathrm{~s}^{-1}$. Bottom: $\mathrm{CS}(5 \rightarrow 4)$ emission observed with SEST. The peak is $1.71 \mathrm{~K} \mathrm{~km} \mathrm{~s}^{-1}$.

Table 2. Angular sizes.

\begin{tabular}{lcccc}
\hline \hline Line & $\begin{array}{c}\text { Frequency } \\
(\mathrm{GHz})\end{array}$ & Telescope & $\begin{array}{c}\text { Beam } \\
\left({ }^{\prime \prime}\right)\end{array}$ & $\begin{array}{c}\text { Obs. size } \\
\left({ }^{\prime \prime}\right)\end{array}$ \\
\hline $\mathrm{CS}(7 \rightarrow 6)$ & 342.883 & APEX & 18 & $24.8 \pm 0.8$ \\
$\mathrm{HCO}^{+}(4 \rightarrow 3)$ & 356.734 & APEX & 17 & $30.7 \pm 1.6$ \\
$\mathrm{H}^{13} \mathrm{CO}^{+}(4 \rightarrow 3)$ & 346.999 & APEX & 18 & $25.5 \pm 0.9$ \\
$\mathrm{CS}(5 \rightarrow 4)$ & 244.936 & SEST & 22 & $38.3 \pm 1.3$ \\
$\mathrm{SO}\left(6_{5} \rightarrow 54\right)$ & 219.949 & SEST & 24 & $35.0 \pm 1.1$ \\
$\mathrm{CS}(2 \rightarrow 1)$ & 97.981 & SEST & 52 & $68.2 \pm 2.6$ \\
$\mathrm{HCO}^{+}(1 \rightarrow 0)$ & 89.189 & NANTEN & 204 & $334 \pm 18$ \\
\hline
\end{tabular}

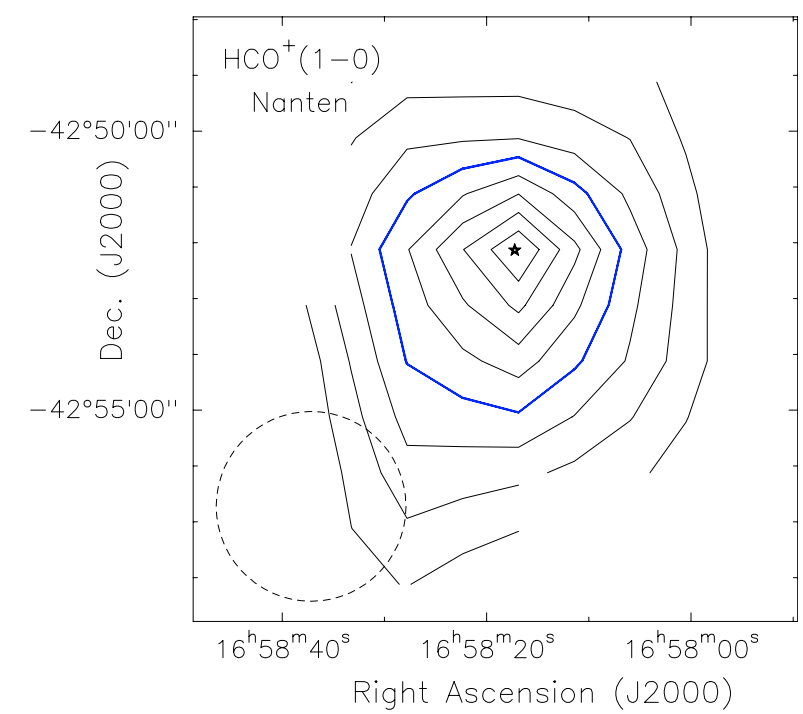

Fig. 2. Map of the velocity integrated $\mathrm{HCO}^{+}(1 \rightarrow 0)$ emission from the ambient gas of IRAS $16547-4247$ observed with NANTEN. Contour levels are $20,30,40,50,60,70,80$, and $90 \%$ of the peak velocity integrated antenna temperature of $6.56 \mathrm{~K} \mathrm{~km} \mathrm{~s}^{-1}$. The $F W H M$ beam is shown in the lower left corner. The star denotes the position of the thermal radio jet.

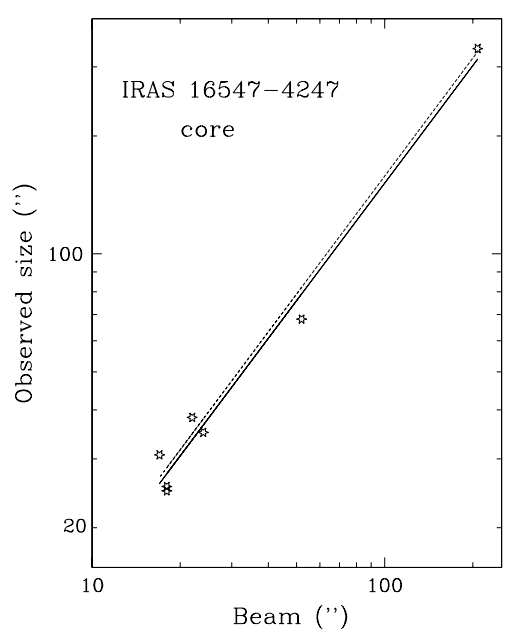

Fig. 3. Observed size versus beam size relationship for the molecular emission from the ambient gas of IRAS 16547-4247. The continuous line corresponds to a linear least squares fit to the data. The dotted line corresponds to the theoretical relation expected for a core with $I \propto r_{\mathrm{p}}^{-1}$.

\subsection{Molecular outflow}

Figure 5 shows the ${ }^{12} \mathrm{CO}(3 \rightarrow 2)$ spectra across a $100^{\prime \prime} \times 160^{\prime \prime}$ region of IRAS 16547-4247, mapped with APEX. Strikingly seen in this figure is the presence, toward the north, of strong emission at velocities redshifted with respect to the systemic ambient cloud velocity of $\sim-30.6 \mathrm{~km} \mathrm{~s}^{-1}$, and toward the south the presence of strong emission at blueshifted velocities. The emission in the redshifted wing is clearly seen in the LSR velocity range from about -22 to $0.8 \mathrm{~km} \mathrm{~s}^{-1}$, and the emission in the blueshifted wing is clearly seen in the range from about -60 to $-38 \mathrm{~km} \mathrm{~s}^{-1}$.

Figure 6 shows the emission in the ${ }^{12} \mathrm{CO}(3 \rightarrow 2)$ line wings integrated over the velocity range $-60<v_{\text {lsr }}<-38 \mathrm{~km} \mathrm{~s}^{-1}$ (continuous lines) and $-22<v_{\mathrm{lsr}}<0.8 \mathrm{~km} \mathrm{~s}^{-1}$ (dashed lines). Clearly distinguished are two lobes symmetrically placed about the thermal radio continuum source (marked with a star). The bulk of the emission at blueshifted velocities with respect to the ambient 


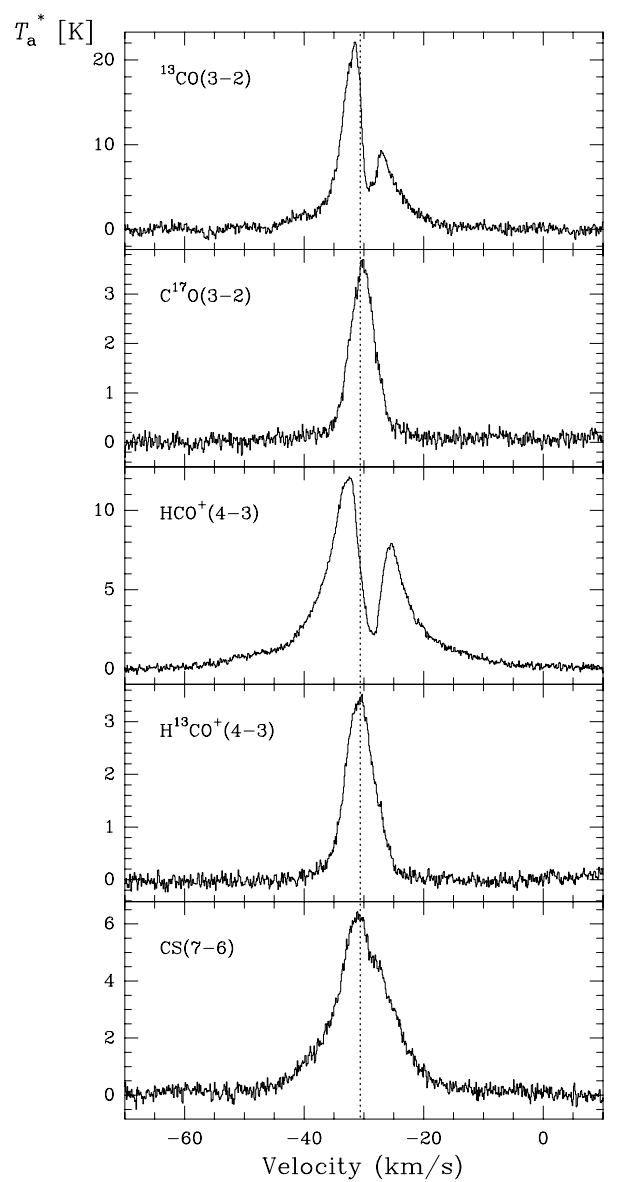

Fig. 4. Spectra of molecular line emission observed with APEX toward the peak position of the IRAS 16547-4247 core. Transitions are given in the upper left corner of the spectra. The vertical dotted line indicates the systemic (ambient) velocity of the gas of $-30.6 \mathrm{~km} \mathrm{~s}^{-1}$.

cloud velocity arises from the south lobe, whereas the bulk of the emission at redshifted velocities arises from the north lobe. Consequently, we will refer to the south lobe as the blue lobe and to the north lobe as the red lobe. The south lobe has FWHM major and minor axis of $46^{\prime \prime}$ and $22^{\prime \prime}$, respectively. The north lobe has $F W H M$ major and minor axis of $51^{\prime \prime}$ and $27^{\prime \prime}$, respectively. The linear extensions in the direction of the flow of the south and north lobes are then 0.65 and $0.72 \mathrm{pc}$, respectively. The position angle of the molecular outflow is $\sim 174^{\circ}$, a value close to that of the $\mathrm{H}_{2}$ flow of $\sim 169^{\circ}$ (determined from Fig. 1 of Brooks et al. 2003). The symmetry axis of the molecular lobes and radio continuum lobes are not exactly aligned, suggesting that the outflow source might be precessing. From the observed major and minor axes, $a$ and $b$, we estimate that the semi-opening angle $(=\arctan (b / 2 a))$ of the blue and red lobes are $13^{\circ} \pm 3^{\circ}$ and $15^{\circ} \pm 3^{\circ}$, respectively. We will adopt a semi-opening angle for the outflow of $14^{\circ}$. The collimation factor is high, with a lengthto-width ratio of 3.7 in the lobes, as determined from the halfpower contour. Figure 7 shows a position-velocity diagram of the ${ }^{12} \mathrm{CO}$ emission along the major axis of the outflow.

\section{Analysis and discussion}

\subsection{Physical properties and kinematics of the massive and dense core}

The observed correlation between the apparent size of the core and the beam size suggests that the underlying specific intensity profile decreases as a power law of distance from the center of the core (e.g., Adams 1991; Ladd et al. 1991). From numerical computations we find that for a source with an intensity profile $I \propto r_{\mathrm{p}}^{-\alpha}$, where $r_{\mathrm{p}}$ is the projected radius, the $F W H M$ apparent size of the observed emission (i.e., the specific intensity convolved with the instrumental beam) is related to the beam size by the expression $\theta_{\text {obs }}=c \theta_{\text {beam }}$, where $c$ is $1.9,1.6$, and 1.2 for $\alpha=0.8,1.0$, and 1.5 , respectively. The observed relationship for IRAS 16547-4247 implies, therefore, that $\alpha=1.0$. Even though the observed relationship was based on observations of different molecular species and lines, the use of the above expression is appropriate because the proportionality factor is independent of the actual value of the intensity.

A power law dependence of the intensity with radial distance most likely indicates that the physical properties of the core are not homogeneous, that is, where the density or temperature change with distance from the center. An analytical expression for the relation between the power law index of molecular line emission intensity and single power law indices of the density, $p$, and temperature, $q$, has not yet been reported, however. At the high densities of the core - with an average density of $5 \times 10^{5} \mathrm{~cm}^{-3}$ (Garay et al. 2003) - we expect that the dust and gas be thermally coupled, and therefore that the density and temperature distributions of the two components be the same. Hence, we will use the expression $\alpha=p+q-1$, appropriate for optically thin dust continuum emission (Motte $\&$ André 2001). The IRAS $16547-4247$ core is heated by a luminous energy source embedded at its central position, hence its temperature is expected to decrease with distance following a power-law with an index of 0.4 (van der Tak et al. 2000). Adopting $q=0.4$, we then infer that the IRAS 16547-4247 massive and dense core is highly centrally condensed, having a power-law density distribution with an index of 1.6.

The characteristics of the line profiles observed toward IRAS 16547-4247 suggest that the molecular gas is undergoing infalling motions. Using the simple model of contracting clouds of Myers et al. (1996), it is possible to derive an infall velocity from the observed velocity difference between the red and blue peaks and the observed brightness temperature of the red peak, blue peak and dip of an optically thick line (see their Eq. (9)). From the spectra of the optically thick $\mathrm{HCO}^{+}(4 \rightarrow 3)$ line and the line width of the optically thin $\mathrm{H}^{13} \mathrm{CO}^{+}(4 \rightarrow 3)$ line (of $5.8 \mathrm{~km} \mathrm{~s}^{-1}$ ) we estimate an inward velocity of $0.5 \pm 0.1 \mathrm{~km} \mathrm{~s}^{-1}$ at a radius of $0.22 \mathrm{pc}$. The latter is an effective radius derived from the map of the $\mathrm{HCO}^{+}(4 \rightarrow 3)$ emission. From the spectra of the optically thick ${ }^{13} \mathrm{CO}(3 \rightarrow 2)$ line and the line width of the optically thin $\mathrm{C}^{17} \mathrm{O}(3 \rightarrow 2)$ line $\left(\right.$ of $\left.5.0 \mathrm{~km} \mathrm{~s}^{-1}\right)$ we estimate an inward velocity of $1.0 \pm 0.1 \mathrm{~km} \mathrm{~s}^{-1}$. The difference in the derived inward speeds could be due to dissimilar contributions of the outflowing gas to the line emission or due to differences in the radial distance of the probed gas due to the different optical depths of the $\mathrm{HCO}^{+}(4 \rightarrow 3)$ and ${ }^{13} \mathrm{CO}(3 \rightarrow 2)$ lines. We note that the inward speeds are significantly smaller than the free-fall velocity expected for a cloud with a total mass of $\sim 1 \times 10^{3} M_{\odot}$ at its outer envelope radius of $0.2 \mathrm{pc}$, suggesting that the core is slowly contracting.

The mass infall rate can be estimated as $\dot{M}_{\text {in }}=4 \pi \mu m_{\mathrm{H}} r^{2} n v_{\text {in }}$, where $n$ and $\mathrm{v}_{\text {in }}$ are the molecular density and infall speed at radius $r$, respectively. Using the values derived from the $\mathrm{HCO}^{+}(4 \rightarrow 3)$ observations $\left(v_{\text {in }}=0.5 \mathrm{~km} \mathrm{~s}^{-1}, r=0.22 \mathrm{pc}\right)$ and the molecular density derived from the dust observations $\left(n=6.7 \times 10^{5} \mathrm{~cm}^{-3}\right.$, Garay et al. 2003), we obtain a mass infall rate of $1 \times 10^{-2} M_{\odot} \mathrm{yr}^{-1}$. Another estimate of the mass infall rate can be obtained assuming that $\dot{M}_{\text {in }}=M / t_{\text {cross }}$, where $M$ 


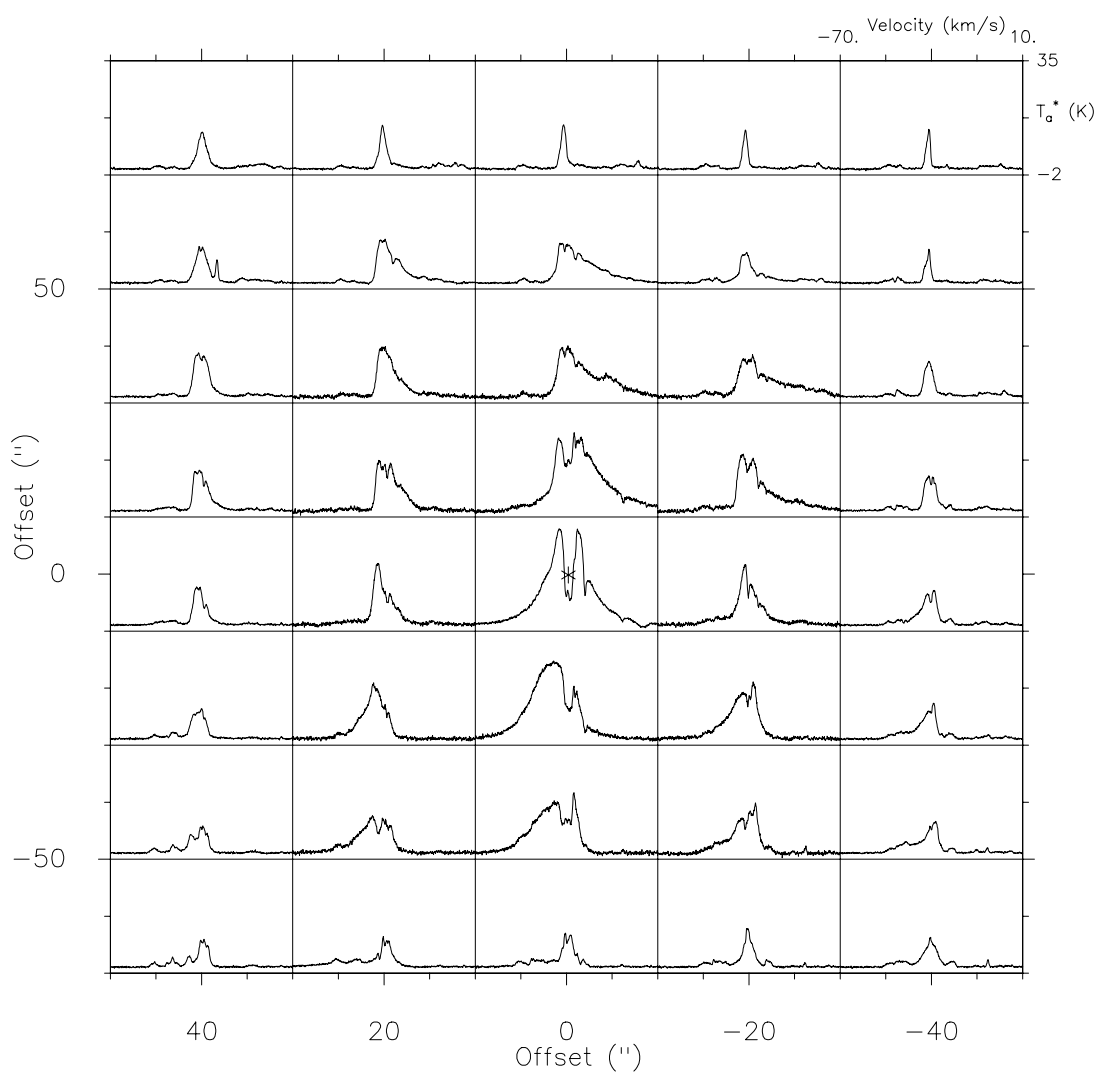

Fig. 5. ${ }^{12} \mathrm{CO}(3 \rightarrow 2)$ spectra over the $100^{\prime \prime}$ by 160 " region mapped with APEX. The asterisk marks the position of the thermal jet. The angular separation between panels is $20^{\prime \prime}$. The velocity scale in each panel runs from -70 to $10 \mathrm{~km} \mathrm{~s}^{-1}$, and the temperature scale from -2 to $35 \mathrm{~K}$.

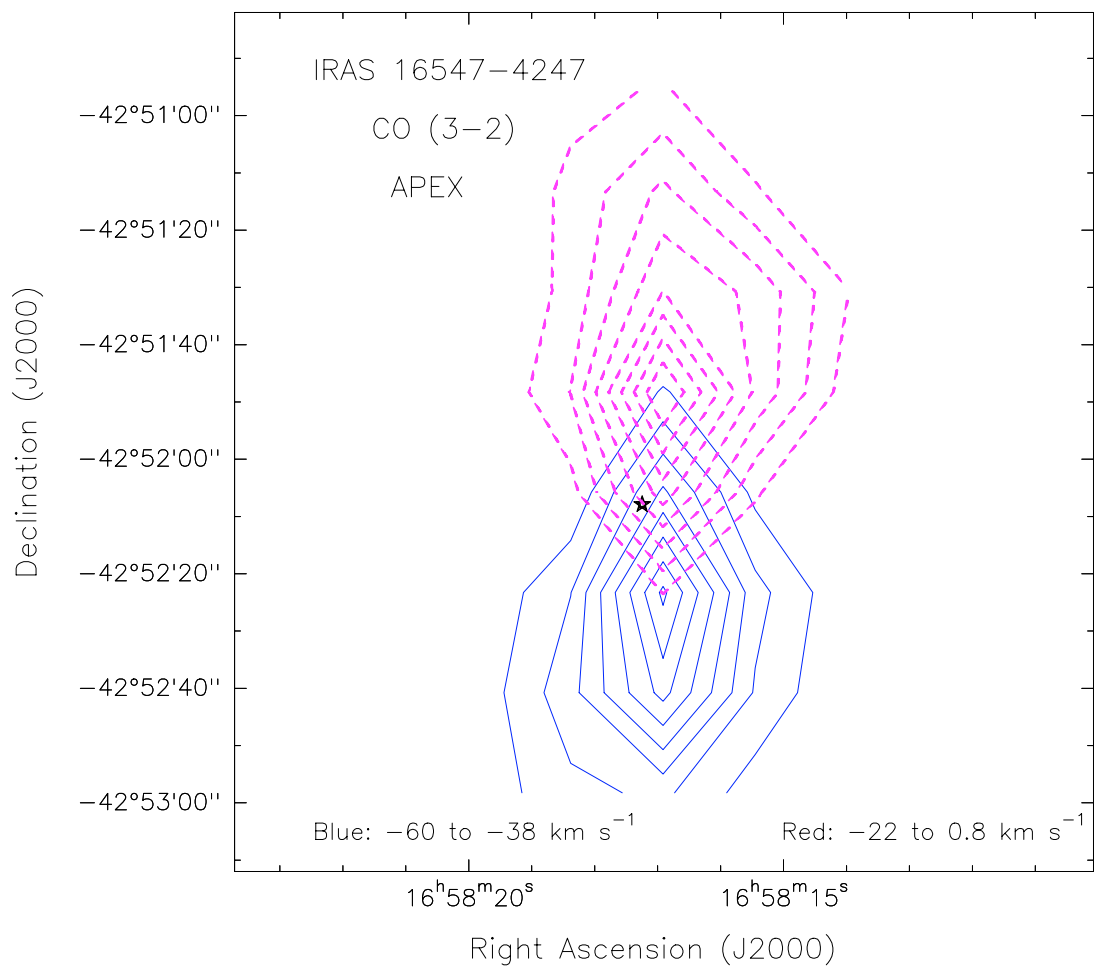

Fig. 6. Contour maps of the ${ }^{12} \mathrm{CO}(3 \rightarrow 2)$ velocity integrated line wing emission from the IRAS $16547-4247$ bipolar outflow. Continuous lines represent emission integrated over the velocity range $-60<v_{\mathrm{lsr}}<-38 \mathrm{~km} \mathrm{~s}^{-1}$, which is blue shifted with respect to the ambient velocity of $-30.6 \mathrm{~km} \mathrm{~s}^{-1}$, and dashed lines emission integrated over the velocity range $-22<v_{\mathrm{lsr}}<0.8 \mathrm{~km} \mathrm{~s}^{-1}$, which is red shifted with respect to the ambient velocity. The star marks the position of the radio continuum jet (Garay et al. 2003). Contour levels are 20, 30, 40, 50, 60, 70, 80, and 90\% of the peak emission of $233 \mathrm{~K} \mathrm{~km} \mathrm{~s}^{-1}$ and $208 \mathrm{~K} \mathrm{~km} \mathrm{~s}^{-1}$ in the blueshifted and redshifted lobes, respectively. 


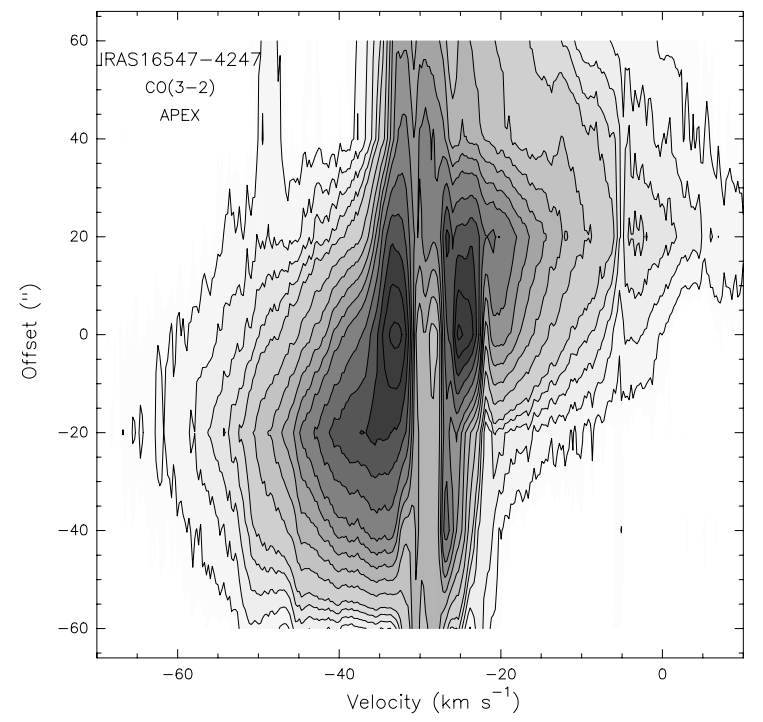

Fig. 7. Position-velocity diagram of the ${ }^{12} \mathrm{CO}(3 \rightarrow 2)$ emission along the major axis of the outflow. Contour levels are 1, 2, 3, 4, 5, 7, 9, 11, $13,15,18,21,24,27$ and $30 \mathrm{~K}$ in $T_{a}^{*}$.

is the core mass $\left(1.3 \times 10^{3} M_{\odot}\right)$ and $t_{\text {cross }}$ the crossing time of the infalling zone at the infall speed. Using $v_{\text {in }}=0.5 \mathrm{~km} \mathrm{~s}^{-1}$ and $r=0.22 \mathrm{pc}$, give $t_{\text {cross }}=4.3 \times 10^{5} \mathrm{yr}$ and $\dot{M}_{\text {in }}=3 \times 10^{-3} M_{\odot} \mathrm{yr}^{-1}$. We emphasize that these are rough estimates and are uncertain by at least a factor of 3 .

\subsection{Molecular outflow}

\subsubsection{Outflow inclination}

A comparison of the observed spatial distribution and positionvelocity diagram of the outflowing gas from IRAS 16547-4247 with synthetic maps of the ${ }^{12} \mathrm{CO}$ line emission, computed for accelerated and constant velocity bipolar outflows (Cabrit \& Bertout 1986, 1990), suggests that this molecular outflow can be approximated, at first order, as flowing with a velocity that is nearly independent of the radial distance from the apex. In what follows we assume that the IRAS 16547-4247 outflow can be modeled by a biconical flow with constant total velocity and derive the values of the model parameters imposed by the observations. First, from the ratio of the observed maximum blueshifted and redshifted velocities within a single lobe, it is possible to estimate the inclination angle, $i$, of the flow (Cabrit et al. 1988). From the maximum values of the absolute magnitude of the blueshifted and redshifted velocities observed toward the blue lobe, of $38.4 \mathrm{~km} \mathrm{~s}^{-1}$ and $13.7 \mathrm{~km} \mathrm{~s}^{-1}$ respectively, and the semi-opening angle of $14^{\circ}$, we derive $i=83^{\circ} \pm 2^{\circ}$. Similarly, from the maximum values of the absolute magnitude of the redshifted and blueshifted velocities observed toward the red lobe, of 43.8 and $22.5 \mathrm{~km} \mathrm{~s}^{-1}$ respectively, we find that $i=85^{\circ} \pm 2^{\circ}$. In both of these derivations we did not consider the emission observed towards the central position. Hereafter, we will adopt an inclination angle for the IRAS $16547-4247$ outflow of $84^{\circ} \pm 2^{\circ}$. Support for a near edge-on configuration is further provided by the presence of blueshifted gas extending along the flow axis into the red lobe and redshifted gas extending along the flow axis into the blue lobe at lower levels than those plotted in Fig. 6. In fact, observations of the $\mathrm{CO}(4-3)$ emission made with APEX with an angular resolution of $13^{\prime \prime}$, to be reported in a forthcoming paper, clearly show that this is the case over a scale greater than the beam size. The flow velocity can then be estimated as $V_{\mathrm{m}} / \cos \left(i-\theta_{\max }\right)$, where $V_{\mathrm{m}}$ is the maximum observed flow velocity. Using for $V_{\mathrm{m}}$ the average of the values observed toward the red and blue lobes, of $41.1 \mathrm{~km} \mathrm{~s}^{-1}$, and the derived value for $i-\theta_{\max }$ of $70^{\circ}$, we derive a flow velocity, $V_{\mathrm{o}}$, of $\sim 120 \mathrm{~km} \mathrm{~s}^{-1}$.

\subsubsection{Physical parameters}

To compute physical parameters of the outflowing gas we followed the standard formalism described in Bourke et al. (1997). A general discussion of the sources of errors have been given by Margulis \& Lada (1985) and Cabrit \& Bertout (1990). The main source of error arises from the difficulty in determining the contribution to the outflow of the emission in the velocity range of the ambient cloud. For the IRAS 16547-4247 outflow, we will adopt as velocity boundary between the blue and red wing emission and the ambient line the values of -38 and $-22 \mathrm{~km} \mathrm{~s}^{-1}$, respectively.

Using expression (A13) of Bourke et al. (1997) we estimate, from the observed wing emission in the velocity range $-60<$ $v_{\text {lsr }}<-38 \mathrm{~km} \mathrm{~s}^{-1}$ for the south lobe and $-22<v_{\text {lsr }}<0.8 \mathrm{~km} \mathrm{~s}^{-1}$ for the north lobe, masses of 7.6 and $8.5 M_{\odot}$ for the south and north lobes, respectively. We assumed an $\left[\mathrm{H}_{2} / \mathrm{CO}\right]$ abundance ratio of $10^{4}$ an that the excitation temperature of the outflowing gas, $T_{\text {ex }}$, is $50 \mathrm{~K}$ (e.g., Beuther et al. 2002a; Leurini et al. 2006). To estimate the contribution to the flow mass from the low velocity material emitting in the same velocity range as the ambient cloud gas we followed the prescription of Margulis \& Lada (1985). Using expression (A16) of Bourke et al. (1997) we estimated that the mass of the low velocity flow within the south and north lobes are 6.1 and $6.4 M_{\odot}$, respectively. We emphasize that the adopted correction is somewhat arbitrary (e.g., Cabrit \& Bertout 1992) introducing here a factor of 2 uncertainty in the flow mass and other flow parameters.

From the observations of the ${ }^{12} \mathrm{CO}(3 \rightarrow 2)$ and ${ }^{13} \mathrm{CO}(3 \rightarrow$ 2) lines it is possible to estimate the opacities of the flowing gas and therefore to assess whether or not our assumption that the ${ }^{12} \mathrm{CO}(3 \rightarrow 2)$ wing emission is optically thin is correct. Using expression (A2) of Bourke et al. (1997), and assuming $T_{\mathrm{ex}}=50 \mathrm{~K}$ and $a=50$, we find that the average ${ }^{12} \mathrm{CO}(3 \rightarrow 2)$ opacity of the blueshifted gas (velocity range between -47.4 to $-38.1 \mathrm{~km} \mathrm{~s}^{-1}$ ) toward the south lobe is $4.1 \pm 1.1$, whereas the average optical depth of the redshifted gas (velocity range between -21.9 to $-12.3 \mathrm{~km} \mathrm{~s}^{-1}$ ) toward the north lobe is $3.5 \pm 2.1$, indicating that the flowing gas is moderately optically thick. To correct for this effect, we multiply the mass determined from the optically thin assumption by the factor $\tau_{\mathrm{w}} /\left(1-\mathrm{e}^{-\tau_{\mathrm{w}}}\right)$, where $\tau_{\mathrm{w}}$ is the average ${ }^{12} \mathrm{CO}(3 \rightarrow 2)$ optical depth in the line wing. We find that the opacity corrected masses within the blue and red lobes are $\sim 32$ and $\sim 31 M_{\odot}$, respectively. The opacity corrected masses for the low velocity material hidden in the ambient line emission are 25 and $23 M_{\odot}$, respectively. Adding the high velocity masses to the low velocity masses, we estimate masses of $\sim 57$ and $54 M_{\odot}$ for the south and north lobes, respectively.

Using the standard LTE formalism it is also possible to estimate the momentum, $P$, the kinetic energy, $E_{\mathrm{k}}$, and the mechanical luminosity, $L_{\mathrm{m}}$, in the flow (Eqs. (A17) of Bourke et al. 1997). These equations assume no correction for flow inclination, and so are strict lower limits. Another method to compute the physical parameters of the outflowing gas is to assume that 
Table 3. Outflow parameters.

\begin{tabular}{|c|c|c|c|c|c|c|c|c|}
\hline \multirow[t]{2}{*}{ Lobe } & \multirow{2}{*}{$\begin{array}{l}M_{\text {total }} \\
\left(M_{\odot}\right) \\
\end{array}$} & $P^{\min }$ & $P^{\max }$ & $E_{k}^{\min }$ & $E_{k}^{\max }$ & $L_{\mathrm{m}}^{\min }$ & $L_{\mathrm{m}}^{\max }$ & \multirow{2}{*}{$\begin{array}{c}t_{\mathrm{d}} \\
(\mathrm{yr})\end{array}$} \\
\hline & & \multicolumn{2}{|c|}{$\left(M_{\odot} \mathrm{km} \mathrm{s}^{-1}\right)$} & \multicolumn{2}{|c|}{$\left(M_{\odot} \mathrm{km}^{2} \mathrm{~s}^{-2}\right)$} & \multicolumn{2}{|r|}{$\left(L_{\odot}\right)$} & \\
\hline South & 57 & $1.4 \times 10^{2}$ & $6.8 \times 10^{3}$ & $1.0 \times 10^{3}$ & $4.1 \times 10^{5}$ & 4.9 & $1.3 \times 10^{4}$ & $5.3 \times 10^{3}$ \\
\hline North & 54 & $1.6 \times 10^{2}$ & $6.5 \times 10^{3}$ & $1.3 \times 10^{3}$ & $3.9 \times 10^{5}$ & 6.1 & $1.1 \times 10^{4}$ & $5.9 \times 10^{3}$ \\
\hline Total & 111 & $3.0 \times 10^{2}$ & $1.3 \times 10^{4}$ & $2.3 \times 10^{3}$ & $8.0 \times 10^{5}$ & 11. & $2.3 \times 10^{4}$ & $\ldots$ \\
\hline
\end{tabular}

all the mass is flowing at a velocity characteristic of the entire flow, $V_{\text {char }}$. The flow parameters are, in this approach, given by

$P=M V_{\text {char }}, E_{\mathrm{k}}=\frac{1}{2} M V_{\text {char }}^{2}, L_{\mathrm{m}}=\frac{1}{2 R} M V_{\text {char }}^{3}$,

where $M$ and $R$ are the total mass and the length of the outflow lobe. Since for the IRAS 16547-4247 outflow the radial velocity with respect to the cone apex is approximately constant at $120 \mathrm{~km} \mathrm{~s}^{-1}$ (Sect. 4.2.1), we will assume $V_{\text {char }}=V_{\mathrm{o}}$, and the parameters determined in this manner are upper limits (Cabrit \& Bertout 1990). In Table 3 we give the parameters of the blue and red lobes of the IRAS 16547-4247 flow calculated using both methods. The dynamical time scale of the outflow, $t_{\mathrm{d}}$, is found from $R / V_{\mathrm{o}}$, and is estimated at $\sim 6 \times 10^{3} \mathrm{yr}$. The geometric mean values for the outflow parameters are $\bar{P} \sim 2.0 \times 10^{3} M_{\odot} \mathrm{km} \mathrm{s}^{-1}$, $\bar{E}_{k} \sim 9 \times 10^{47} \mathrm{erg}\left(\right.$ or $4.3 \times 10^{4} M_{\odot} \mathrm{km}^{2} \mathrm{~s}^{-2}$ ), and $\bar{L}_{\mathrm{m}} \sim$ $5.0 \times 10^{2} L_{\odot}$. The mass outflow rate is $\sim 2 \times 10^{-2} M_{\odot} \mathrm{yr}^{-1}$ and the momentum outflow rate is $\sim 0.3 M_{\odot} \mathrm{km} \mathrm{s}^{-1} \mathrm{yr}^{-1}$.

Shepherd \& Churchwell (1996) and Beuther et al. (2002c) found that the correlations between physical parameters derived for outflows driven by low-mass protostars can be extended to the high-mass regime. The derived value of the physical parameters of the IRAS 16547-4247 outflow are characteristic of molecular flows driven by high-mass young stellar objects. The derived values of the mass entrainment rate and mechanical force of the IRAS 16547-4247 flow indicate that this is one of the most powerful outflows known (see Figs. 4 and 5 of Beuther et al. 2002c), even if no correction is made for material hidden at the cloud velocity. In particular, the correlations observed between these parameters and the bolometric luminosity of the driving source predicts that the IRAS $16547-4247$ outflow is being driven by a source with a luminosity of $\sim 1 \times 10^{5} L_{\odot}$. The total far-infrared luminosity of the IRAS 16547-4247 core, computed using the IRAS fluxes (see Casoli et al. 1986), is $\sim 6.2 \times$ $10^{4} L_{\odot}$. This strongly suggest that the bulk of the luminosity of IRAS $16547-4247$ is due to a single luminous object which drives a bipolar outflow and a highly collimated thermal jet. We suggest that at the center of IRAS 16547-4247 lies a very young massive protostar which is still accreting matter, building up its mass from a surrounding infalling envelope. The mass accretion rate is large enough, $\sim 1 \times 10^{-2} M_{\odot} \mathrm{yr}^{-1}$ to prevent the development of an observable UC H II region (Yorke 1984; Walmsley 1995). Ours and previously published results show that IRAS 16547-4247 harbors a massive and young molecular outflow, a thermal jet (Brooks et al. 2003) and a rotating disk-like structure (Franco-Hernández et al. 2005), making it the most luminous embedded YSO known in the Galaxy that exhibits these three phenomena (see Table 3 of Garay \& Lizano 1999).

\section{Conclusions}

We have observed the IRAS 16547-4247 massive and dense core in the $J=3 \rightarrow 2$ transitions of ${ }^{12} \mathrm{CO}$ and ${ }^{13} \mathrm{CO}, J=4 \rightarrow 3$ transitions of $\mathrm{HCO}^{+}$and $\mathrm{H}^{13} \mathrm{CO}^{+}$, and $J=7 \rightarrow 6$ transition of $\mathrm{CS}$, with angular resolution of $\sim 18^{\prime \prime}$. The main results and conclusions presented in this paper are summarized as follows.

1. The observations of the ${ }^{12} \mathrm{CO}(3 \rightarrow 2)$ line emission reveal the presence of a collimated bipolar outflow with lobes extending $\sim 0.7 \mathrm{pc}$ in opposite directions from the thermal jet located at the center of the core. The bulk of the emission from the south lobe arises at velocities that are blueshifted (velocity range from about -60 to $-38 \mathrm{~km} \mathrm{~s}^{-1}$ ) with respect to the ambient cloud velocity of $-30.6 \mathrm{~km} \mathrm{~s}^{-1}$, while the bulk of the emission from the north lobe arises from velocities that are redshifted (velocity range from about -22 to $0.8 \mathrm{~km} \mathrm{~s}^{-1}$ ).

2. The observed morphology and velocity structure of the flow is well accounted for with a simple model of a biconical outflow with a semi-opening angle of $14^{\circ}$, in which the gas moves outwards with a constant radial velocity (with respect to the cone apex) of $\sim 120 \mathrm{~km} \mathrm{~s}^{-1}$ and that the outflow axis is inclined from the line of sight at an angle of $\sim 84^{\circ}$.

3. The total flow masses within each lobe, taking into account the mass in the velocity range of the ambient cloud and optical depth effects of the flowing gas, are $57 M_{\odot}$ and $54 M_{\odot}$ for the south and north lobes, respectively. The molecular outflow has a mass outflow rate of $\sim 2 \times 10^{-2} M_{\odot} \mathrm{yr}^{-1}$, a momentum of $\sim 2 \times 10^{3} M_{\odot} \mathrm{km} \mathrm{s}^{-1}$ and a kinetic energy of $\sim 9 \times 10^{47} \mathrm{erg}$. Its dynamical age is $\sim 6 \times 10^{3}$ years and the mechanical luminosity is $\sim 5.0 \times 10^{2} L_{\odot}$.

4. The molecular line emission from the ambient gas arises from nearly circular structures. The observed angular sizes depend, however, on the beam size of the observations. The observed relation $\left(\theta_{\text {obs }}=1.59 \theta_{\text {beam }}\right)$ can be well fitted by a model in which the intensity decreases with projected radial distance following a power-law dependence with an index of $1.0\left(I \propto r_{\mathrm{p}}^{-1.0}\right)$. This in turn implies that the massive and dense core is highly centrally condensed, with the density decreasing with radial distance following a power-law dependence with an index of $1.6\left(n \propto r^{-1.6}\right)$.

5. The characteristics of the line profiles observed toward IRAS 16547-4247 indicate that the molecular gas is undergoing large scale infalling motions. From the observed spectra in the $\mathrm{HCO}^{+}(4 \rightarrow 3)$ and ${ }^{13} \mathrm{CO}(3 \rightarrow 3)$ lines we derived inward speeds of 0.5 and $1.0 \mathrm{~km} \mathrm{~s}^{-1}$, respectively. The mass infall rate is of the order of $10^{-2} M_{\odot} \mathrm{yr}^{-1}$.

6. We conclude that IRAS $16547-4247$ is a massive and dense molecular core in a state of collapse, harboring at its center the most luminous and massive protostellar object known in the Galaxy. The massive YSO is in a very early stage of evolution, being formed under very high mass accretion rates and driving a highly collimated thermal jet and an energetic bipolar molecular outflow.

Acknowledgements. G.G., D.M. and L.B. gratefully acknowledge support from the Chilean Centro de Astrofísica FONDAP No. 15010003. 


\section{References}

Adams, F. C. 1991, ApJ, 382, 544

Beuther, H., Schilke, P., Gueth, F., et al. 2002a, A\&A, 387, 931

Beuther, H., Schilke, P., Menten, K. M., et al. 2002b, ApJ, 566, 945

Beuther, H., Schilke, P., Sridharan, T. K., et al. 2002c, A\&A, 383, 892

Bourke, T. L., Garay, G., Lehtinen, K. K., et al. 1997, ApJ, 476, 781

Bronfman, L., Nyman, L-Å., \& May, J. A. 1996, A\&AS, 115, 81

Brooks, K. J., Garay, G., Mardones, D., \& Bronfman, L. 2003, ApJ, 594, L131

Cabrit, S., \& Bertout, C. 1986, ApJ, 307, 313

Cabrit, S., \& Bertout, C. 1990, ApJ, 348, 530

Cabrit, S., \& Bertout, C. 1992, A\&A, 261, 274

Cabrit, S., Goldsmith, P. F., \& Snell, R. L. 1988, ApJ, 334, 196

Casoli, F., Dupraz, C., Gerin, M., Combes, F., \& Boulanger, F. 1986, A\&A, 169, 281

Churchwell, E. 2000, Unsolved Problems in Stellar Evolution, ed. M. Livio (Cambridge University Press)

Faúndez, S., Bronfman, L., Garay, G., et al. 2004, A\&A, 426, 97

Franco-Hernández, R., Moran, J. M., Rodríguez, L. F., \& Garay, G. 2005, in Protostars and Planets V, Contribution No. 1286

Garay, G., \& Lizano S. 1999, PASP, 111, 1049

Garay, G., Brooks, K., Mardones, D., \& Norris, R. P. 2003, ApJ, 587, 739
Güsten, R., Nyman, L. Å., Schilke, P., et al. 2006, A\&A, 454, L13 Juvela, M. 1996, A\&AS, 118, 191

Ladd, E. F., Adams, F. C., Fuller, G. A., et al. 1991, ApJ, 382, 555

Leurini, S., Schilke, P., Parise, B., et al. 2006, A\&A, 454, L83

Mardones, D., Myers, P. C., Tafalla, M., et al. 1997, ApJ, 489, 719

Margulis, M., \& Lada, C. J. 1985, ApJ, 299, 925

Motte, F., \& André, P. 2001, A\&A, 365, 440

Myers, P. C., Mardones, D., Tafalla, M., Williams, J. P., \& Wilner, D. J. 1996, ApJ, 465, L133

Plume, R., Jaffe, D. T., \& Evans, N. J. II. 1992, ApJS, 78, 505

Plume, R., Jaffe, D. T., Evans, N. J. II., Martín-Pintado, J., \& Gómez-Gonzalez, J. 1997, ApJ, 476, 730

Rodríguez, L. F., Garay, G., Brooks, K. J., \& Mardones, D. 2005, ApJ, 626, 953

Shepherd, D. S., \& Churchwell, E. 1996, ApJ, 472, 225

Shu, F. H., Adams, F. C., \& Lizano, S. 1987, ARA\&A, 25, 23

Snell, R. L., \& Loren, R. B. 1977, ApJ, 211, 122

van der Tak, F. F. S., van Dishoeck, E. F., Evans II, N. J., \& Blake, G. A. 2000, ApJ, 537, 283

Walmsley, C. M. 1995, RMAA Conf. Ser., 1, 137

Yorke, H. W. 1984, Workshop on Star Formation, ed. R. D. Wolstencroft (Edinburgh: Royal Observatory), 63

Zhang, Q., Hunter, T. R., Brand, J., et al. 2001, ApJ, 552, L167 